\title{
Comprehensive analysis of differentially expressed genes reveals the promotive effects of UBE2T on colorectal cancer cell proliferation
}

\author{
MIN LUO ${ }^{1,2}$ and YUQIAN ZHOU ${ }^{1,2}$ \\ ${ }^{1}$ Department of Gastroenterology, The Second Xiangya Hospital; ${ }^{2}$ Research Center of Digestive Disease, \\ Central South University, Changsha, Hunan 410011, P.R. China
}

Received May 17, 2020; Accepted April 1, 2021

DOI: $10.3892 / \mathrm{ol} .2021 .12975$

\begin{abstract}
Colorectal cancer (CRC) is one of the most common malignancies worldwide. Via analysis using The Cancer Genome Atlas database, the present study identified 1,835 genes that were differentially expressed in CRC, including 811 upregulated and 1,024 downregulated genes. Enrichment analyses using the Database for Annotation, Visualization and Integrated Discovery tool revealed that these differentially expressed genes were associated with the regulation of CRC progression by modulating multiple pathways, such as 'Cell Cycle, Mitotic', 'DNA Replication', 'Mitotic M-M/G1 phases' and 'ATM pathway'. To identify the key genes in CRC, protein-protein interaction (PPI) network analysis was performed and the hub modules in upregulated and downregulated PPI networks were identified. Ubiquitin-conjugating enzyme E2 T (UBE2T), a member of the E2 family, was identified to be a key regulator in CRC. To the best of our knowledge, the present study was the first to demonstrate that UBE2T expression was upregulated in CRC samples compared with normal tissues. Kaplan-Meier analysis revealed that higher expression levels of UBE2T were associated with worse prognosis compared with lower UBE2T expression levels in CRC. Additionally, the present study demonstrated that knockdown of UBE2T inhibited CRC cell proliferation. Flow cytometry assays revealed that UBE2T knockdown induced cell cycle arrest at $\mathrm{G}_{1}$ phase and apoptosis in vitro. These results suggested that UBE2T may be a novel potential biomarker for CRC.
\end{abstract}

Correspondence to: Professor Yuqian Zhou, Department of Gastroenterology, The Second Xiangya Hospital, Central South University, 87 Xiangya Road, Changsha, Hunan 410011, P.R. China E-mail: yuqianzhou@csu.edu.cn

Key words: colorectal cancer, differentially expressed genes, ubiquitin-conjugating enzyme $\mathrm{E} 2 \mathrm{~T}$, prognosis, proliferation

\section{Introduction}

Colorectal cancer (CRC) is one of the most common malignancies worldwide (1). In 2017, 46.9 per 100,000 population were newly diagnosed with CRC and there are 17.7 per 100,000 CRC-associated deaths annually in the United States (2). Unhealthy diet, obesity, smoking and genetics may contribute to $\mathrm{CRC}$, which has been a main cause of tumor-related morbidities in China (3). Notably, biomarkers for the prognosis of advanced CRC remain limited. Therefore, it is urgent to elucidate the molecular mechanisms involved in the regulation of CRC development.

Ubiquitin-conjugating enzyme E2 (UBE2) T, a member of the E2 family, serves important roles in modulating cell proliferation, apoptosis and signal transduction (4-6). For instance, UBE2T induces hepatocellular carcinoma growth by enhancing cell cycle progression (6). Additionally, UBE2T has been demonstrated to regulate the Fanconi anemia signaling pathway by modulating Fanconi anemia group D2 protein (FANCD2) monoubiquitination, which is a key step in the DNA damage signaling pathway (7). The upregulation of UBE2T has been reported in multiple cancer types, and is associated with the regulation of cancer cell proliferation and metastasis (8-12), including in gastric (11) and prostate cancer (12). However, the functional roles of UBE2T in CRC remain unclear and require further investigation.

The present study analyzed The Cancer Genome Atlas (TCGA) dataset to identify differentially expressed genes (DEGs) in CRC. Moreover, protein-protein interaction network analysis was performed to identify hub regulators in the progression of CRC. Finally, loss of function assays using short hairpin RNA (shRNA/sh) was performed to confirm the potential functions of hub gene UBE2T in CRC. The present results indicated that UBE2T may be a novel key regulator of CRC progression.

\section{Materials and methods}

Analysis of public CRC RNA-Seq data. The RNA-Seq data from TCGA (http://cancergenome.nih.gov/) was utilized to compare gene expression between CRC and non-tumor tissues. The genes with $\mid \log 2$ [fold change (FC)] $>1.0$ and P-value $<0.05$ used as the threshold values. 
Gene Ontology (GO) and Kyoto Encyclopedia of Genes and Genomes (KEGG) enrichment analyses of DEGs. The Database for Annotation, Visualization and Integrated Discovery (DAVID; version 6.8; https://david.ncifcrf. gov/summary.jsp) provides a comprehensive set of functional annotation tools for investigators to understand the biological meaning behind a number of different genes $(13,14)$. GO functional annotation and KEGG analysis of DEGs was performed and visualized using the ImageGP database (http://www.ehbio.com/ImageGP/index. php/Home/Index/index.html).

PPI network construction and key module identification. The PPI network was built using the Search Tool for the Retrieval of Interacting Genes (STRING; version 10.0) online database (15). Cytoscape (version 3.6.1) is a bioinformatics software for the visualization of molecular interaction networks (16). The Molecular Complex Detection (MCODE) plug-in of Cytoscape was used to find closely connected regions in a network (17). The PPI network was visualized using Cytoscape and the most significant module was identified using MCODE. The selection criteria were as follows: Degree cut-off, 2; node score cut-off, 0.2; max depth, 100; and k-score, 2.

Cell culture. The RKO cell line was purchased from American Type Culture Collection and cultured in DMEM (Gibco; Thermo Fisher Scientific, Inc.) supplemented with 10\% FBS (HyClone; Cytiva) at $37^{\circ} \mathrm{C}$ under a $5 \% \mathrm{CO}_{2}$ atmosphere as previously described (18).

Reverse transcription-quantitative PCR (RT-qPCR). Total RNA was extracted using a TRIzol ${ }^{\circledR}$ kit (Takara Biotechnology Co., Ltd.) from RKO cells. RT was conducted using the PrimeScript RT Reagent kit (Takara Biotechnology Co., Ltd.). The temperature and duration of RT were: $37^{\circ} \mathrm{C}$ For $30 \mathrm{sec}$, followed by $85^{\circ} \mathrm{C}$ for $5 \mathrm{sec}$ and $4^{\circ} \mathrm{C}$ for $10 \mathrm{~min}$. qPCR was performed using SYBR Premix Ex Taq II (Takara Biotechnology Co., Ltd.) according to the manufacturer's protocol. The thermocycling conditions used were as follows: $95^{\circ} \mathrm{C}$ For $30 \mathrm{sec}$, followed by 40 cycles of $95^{\circ} \mathrm{C}$ for $5 \mathrm{sec}$ and $60^{\circ} \mathrm{C}$ for $40 \mathrm{sec}$. The following primer pairs were used for qPCR: UBE2T forward, 5'-ATCCCTCAACATCGCAAC TGT-3' and reverse, 5'-CAGCCTCTGGTAGATTATCAA GC-3'; and GAPDH forward, 5'-TGACTTCAACAGCGA CACCCA-3' and reverse, 5'-CACCCTGTTGCTGTAGCC AAA-3'. GAPDH was used as endogenous control. The relative expression of target genes was calculated using the $2^{-\Delta \Delta C q}$ method (19).

Western blotting. The western blot assay was performed according to a previous report (20). Total protein was extracted from RKO cells using RIPA lysis buffer (Beyotime Institute of Biotechnology). The concentration of proteins was determined using the Bradford method. Cell lysates (30 $\mu \mathrm{g} /$ lane) were separated using 10\% SDS-PAGE and transferred to PVDF membranes (EMD Millipore). Then, the membrane was blocked with 5\% skimmed milk for $1 \mathrm{~h}$ at room temperature. Next, the membrane was incubated with primary antibodies at $4^{\circ} \mathrm{C}$ overnight. The antibodies used in the present study included GAPDH
(1:5,000; cat no. 10494-1-AP; ProteinTech Group, Inc.), $\beta$-actin (1:1,000; cat. no. ab8227; Abcam), Bcl-2 (1:1,000; cat. no. sc-492; Santa Cruz Biotechnology, Inc.), cleaved caspase-3 (1:500; cat. no. 9664; Cell Signaling Technology, Inc.) and UBE2T (1:2,000; cat. no. 10105-2-AP; ProteinTech Group, Inc.). After washing with TBST ( $0.1 \%$ Tween) three times, the membranes were incubated with horseradish peroxidase-conjugated anti-rabbit IgG secondary antibody (1:3,000; cat. no. 7074; Cell Signaling Technology, Inc.) at room temperature for $1 \mathrm{~h}$. Finally, the membrane was visualized using an Immobilon ${ }^{\mathrm{TM}}$ Western Chemiluminescent HRP substrate (EMD Millipore).

Lentivirus vector construction for RNA interference. Recombinant lentiviral vectors were constructed as previously described (21). shRNA against UBE2T (shUBE2T; 5'-GCAACTGTGTTGACCTCTATT-3') and shRNA negative control (shNC; 5'-TTCTCCGAACGTGTCACGT-3') were purchased from Shanghai GeneChem Co., Ltd. The shRNAs were annealed and ligated into the linearized GV115 lentiviral vector (Shanghai GeneChem Co., Ltd.). Next, $10 \mu \mathrm{g}$ pGV115-shControl (Ctrl)/pGV115-shUBE2T were transfected into 293T cells (Sangon Biotech, Co., Ltd.) with the pHelper system (Shanghai GeneChem Co., Ltd.) to produce lentiviral particles. Lentiviruses were harvested after $48 \mathrm{~h}$. Next, RKO cells were infected and cultured in RPMI-1640 medium with lentiviruses at a multiplicity of infection of 10 for $48 \mathrm{~h}$ at $37^{\circ} \mathrm{C}$. At $48 \mathrm{~h}$ after transfection, the transfection efficiency was determined using reverse transcription-quantitative (RT-q) PCR and western blotting.

Cell proliferation analysis. An adherent cell cytometry system, Celigo $^{\circledR}$, was used to detect the proliferation of CRC cells as previously described (21).

Colony formation assays. A total of 1,000 RKO cells were seeded into 6-well plates and cultured with DMEM (Gibco; Thermo Fisher Scientific, Inc.) at $37^{\circ} \mathrm{C}$ with $5 \% \mathrm{CO}_{2}$ for 2 weeks. Subsequently, the colonies were washed with $1 \mathrm{ml}$ PBS and fixed with $1 \mathrm{ml}$ 4\% paraformaldehyde (Beyotime Institute of Biotechnology) for $15 \mathrm{~min}$ at room temperature. Next, the colonies were stained with $500 \mu \mathrm{l}$ Giemsa (Sigma-Aldrich; Merck KGaA) for 15-20 min at room temperature. Finally, the colonies were observed under an inverted light microscope (magnification, x200; IX71; Olympus Corporation) and counted using ImageJ software (version 4.0; National Institutes of Health).

Cell cycle and apoptosis assays. At 2 days after transfection with shUBE2T or shNC lentivirus, RKO cells were collected, washed twice with PBS and resuspended in staining buffer containing $0.03 \%$ Triton X-100. Subsequently, $5 \mu 1$ Annexin V-allophycocyanin (eBioscience; Thermo Fisher Scientific, Inc.) was added to the cells to detect cell apoptosis, while $50 \mathrm{ng} / \mathrm{ml}$ PI (Sigma-Aldrich; Merck KGaA) was added to the cells to analyze the cell cycle. Finally, the effects of UBE2T knockdown on the cell cycle and apoptosis were detected using a FACSCalibur instrument (BD Biosciences). The cell cycle was analyzed using the FACSCalibur flow cytometer (BD Biosciences). 


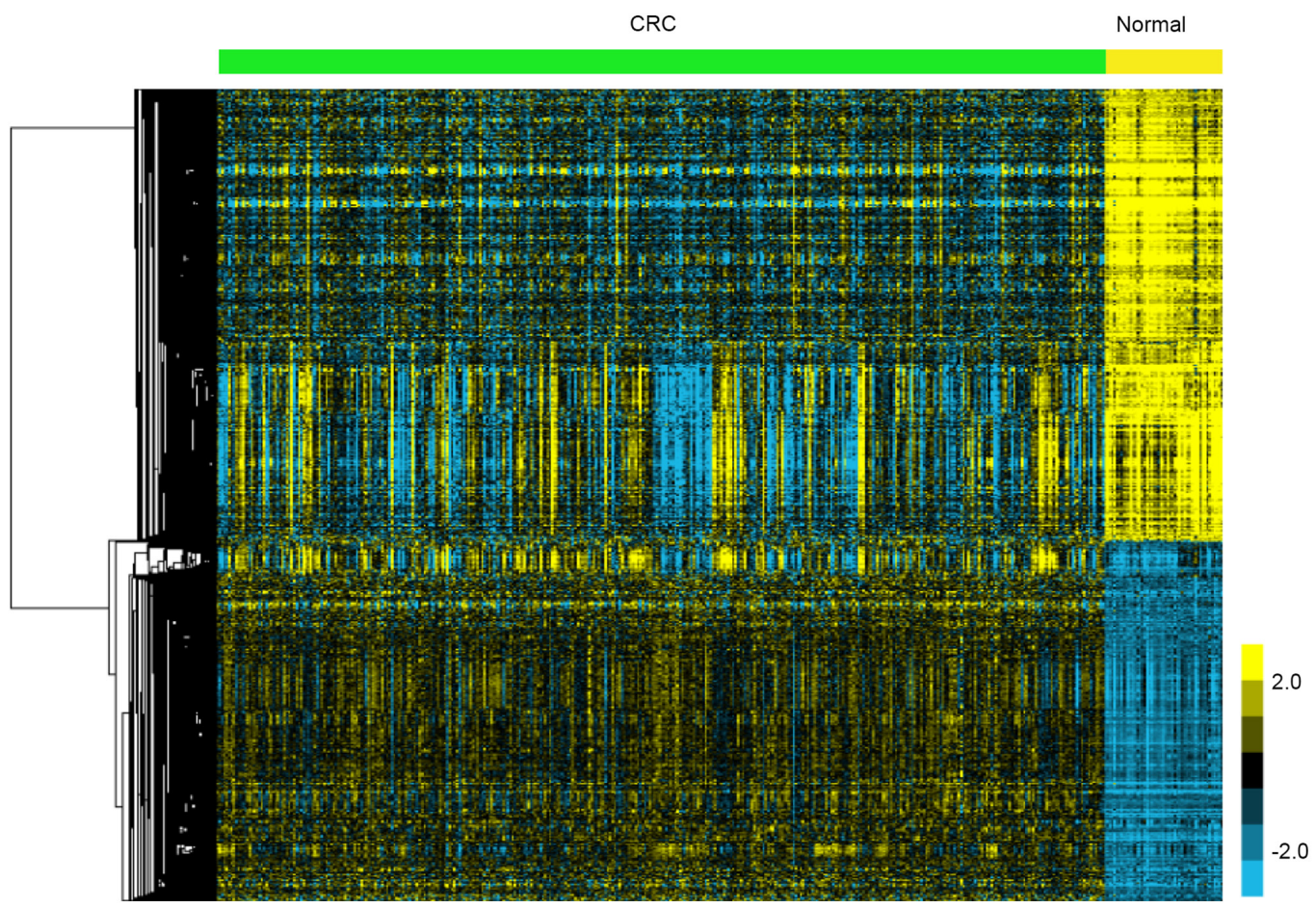

Figure 1. Identification of differentially expressed genes in CRC. Heatmap analysis showed differentially expressed genes in CRC compared with non-tumor tissues. Yellow indicates upregulation and blue indicates downregulation. CRC, colorectal cancer.

Statistical analysis. Statistical analysis was performed using GraphPad Prism v6.0 software (GraphPad Software, Inc.). All assays were performed three times and data are presented as the mean \pm standard deviation. Unpaired Student's t-test was used to compare differences between two groups $\mathrm{P}<0.05$ was considered to indicate a statistically significant difference.

\section{Results}

A total of 811 upregulated and 1,024 downregulated genes were identified in $C R C$. In the present study, TCGA data analysis showed 1,835 genes were identified to be DEGs, including 811 upregulated and 1,024 downregulated genes in CRC compared to adjacent non-tumor tissues. The heatmap of DEGs is presented in Fig. 1.

Enrichment analyses of upregulated and downregulated genes in CRC. To determine the function of the identified DEGs in CRC, gene enrichment analysis was performed using DAVID. Enrichment analyses of upregulated and downregulated genes were performed separately. By subjecting the upregulated genes to enrichment analysis, numerous enriched gene sets were observed. Based on bioinformatics analysis, the top 15 biological processes related to upregulated genes included 'Cell Cycle, Mitotic', 'DNA Replication', 'ATM pathway', 'Mitotic M-M/G1 phases', 'ATR signaling pathway', 'M Phase', 'Mitotic G1-G1/S phases', 'S Phase', 'Mitotic Prometaphase', 'E2F transcription factor network' 'FOXM1 transcription factor network', 'G2/M Checkpoints', 'Activation of ATR in response to replication stress', 'DNA strand elongation' and 'Unwinding of DNA' (Fig. 2A).
By subjecting the downregulated genes to enrichment analysis, numerous enriched gene sets were observed. Based on bioinformatics analysis, the top 15 biological processes related to down-regulated genes included 'integrin family cell surface interactions', ' $\beta 1$ integrin cell surface interactions', 'TRAIL signaling pathway', 'glypican pathway', 'proteoglycan syndecan-mediated signaling events', 'Sphingosine 1-phosphate (S1P) pathway', 'endothelins', ' $\alpha 9 \beta 1$ integrin signaling events', 'glypican 1 network', 'nectin adhesion pathway', 'IL3-mediated signaling events', 'IL5-mediated signaling events', 'GMCSF-mediated signaling events', 'epithelial-to-mesenchymal transition' and 'smooth muscle Contraction' (Fig. 2B).

Construction of upregulated and downregulated PPI networks in CRC. The STRING online database was used to analyze the interactions among the DEGs. The results were extracted and visualized using Cytoscape software. After excluding the isolated nodes, the final upregulated PPI network was composed of 649 nodes and 11,747 edges (Fig. 3). The downregulated PPI network was composed of 771 nodes and 4,232 edges (Fig. 4).

Identification of hub upregulated and downregulated genes in CRC. A significant densely connected module was identified using the MCODE plug-in. The hub upregulated network included 101 nodes (Fig. 5A). Notably, we found multiple ubiquitin-conjugating enzyme $\mathrm{E}$ family members served a key role in this network, including UBE2T and UBE2C (Fig. 5A). The bioinformatics analysis demonstrated that this hub network was associated with 'DNA metabolic process', 'cell cycle $\mathrm{G}_{2} / \mathrm{M}$ phase transition', 'cellular process', 'cell cycle', 'regulation of cyclin-dependent protein kinase activity', 'cell cycle $\mathrm{G}_{1} / \mathrm{S}$ phase transition', 'mitotic cell cycle phase transition', 

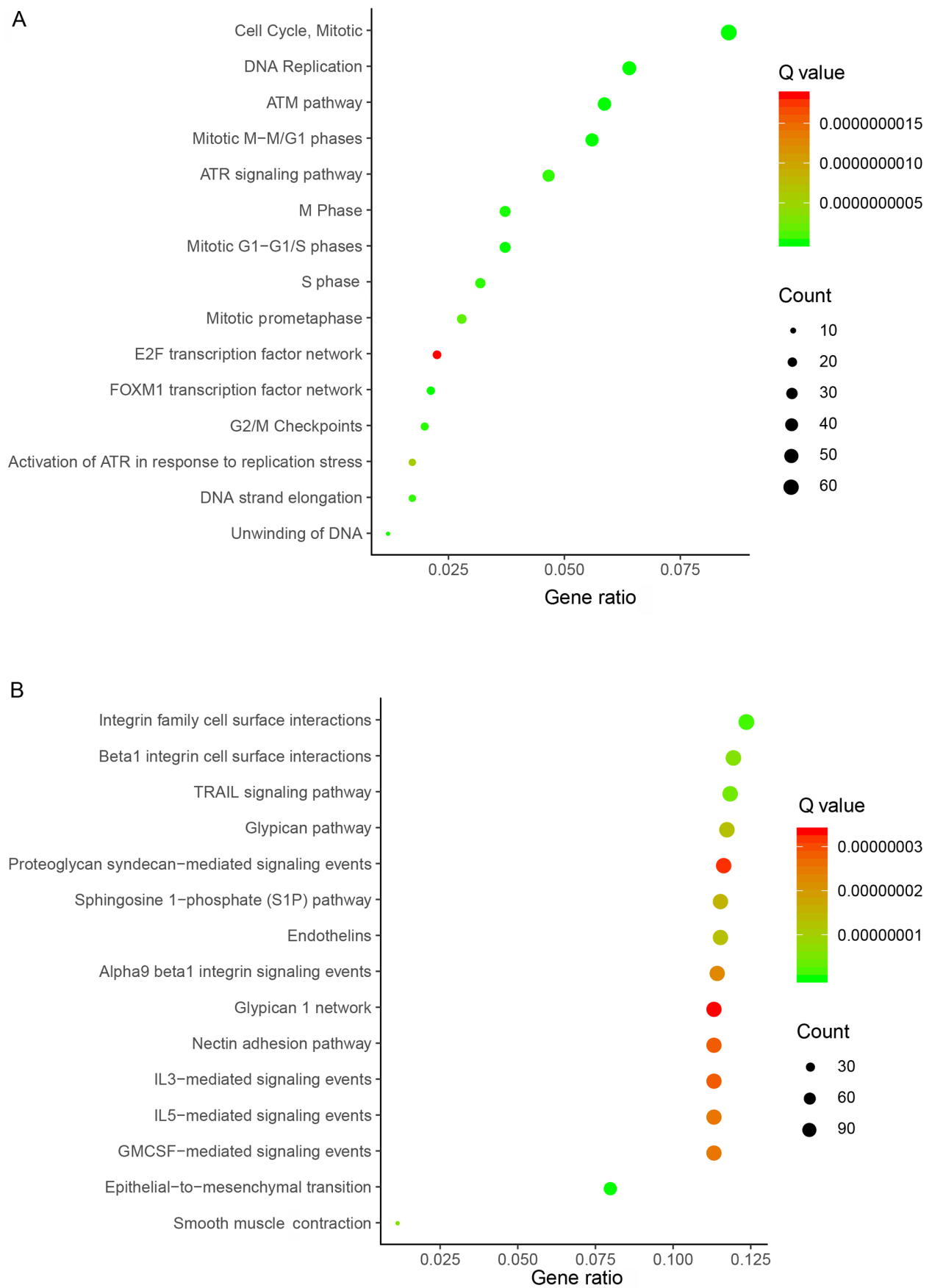

Figure 2. Bioinformatics analysis of differentially expressed genes in colorectal cancer. (A) Top 15 biological processes enriched in upregulated genes. (B) Top 15 biological processes enriched in downregulated genes. ATM, ATM serine/threonine kinase; ATR, ATR serine/threonine kinase; E2F, E2 transcription factor; FOXM1, forkhead box M1; TRAIL, TNF superfamily member 10; S1P, sphingosine-1-phosphate; GMCSF, granulocyte-macrophage colony-stimulating factor.

'regulation of cell cycle', 'mitotic sister chromatid segregation', 'cell proliferation', 'regulation of cell cycle process', 'cell cycle', 'mitotic cell cycle process', 'microtubule-based movement', 'cytokinesis', 'response to radiation', 'single-organism process', 'mitotic nuclear division', 'regulation of cellular process', 'meiotic cell cycle' and 'regulation of microtubule cytoskeleton organization' (Fig. 6A).

The hub downregulated network included 15 nodes (Fig. 5B). The bioinformatics analysis demonstrated that this hub network was associated with 'Chemokine signaling pathway', 'response to external stimulus', 'G-protein coupled receptor signaling pathway', 'cellular response to stimulus', 'regulation of biological quality' and 'negative regulation of cellular processes’ (Fig. 6B).

Higher UBE2T expression in CRC samples is associated with shorter survival time. The present study focused on UBE2T, which had been reported to be a key role in the DNA damage repair pathway via FANCD2 monoubiquitination $(22,23)$. To the best of our knowledge, the molecular functions of UBE2T in CRC remain unclear. In aforementioned bioinformatics analysis, it was reported that UBE2T is a key regulator in hub network 1 (Fig. 5A) and involved in regulating cell proliferation, cell cycle and DNA metabolic 


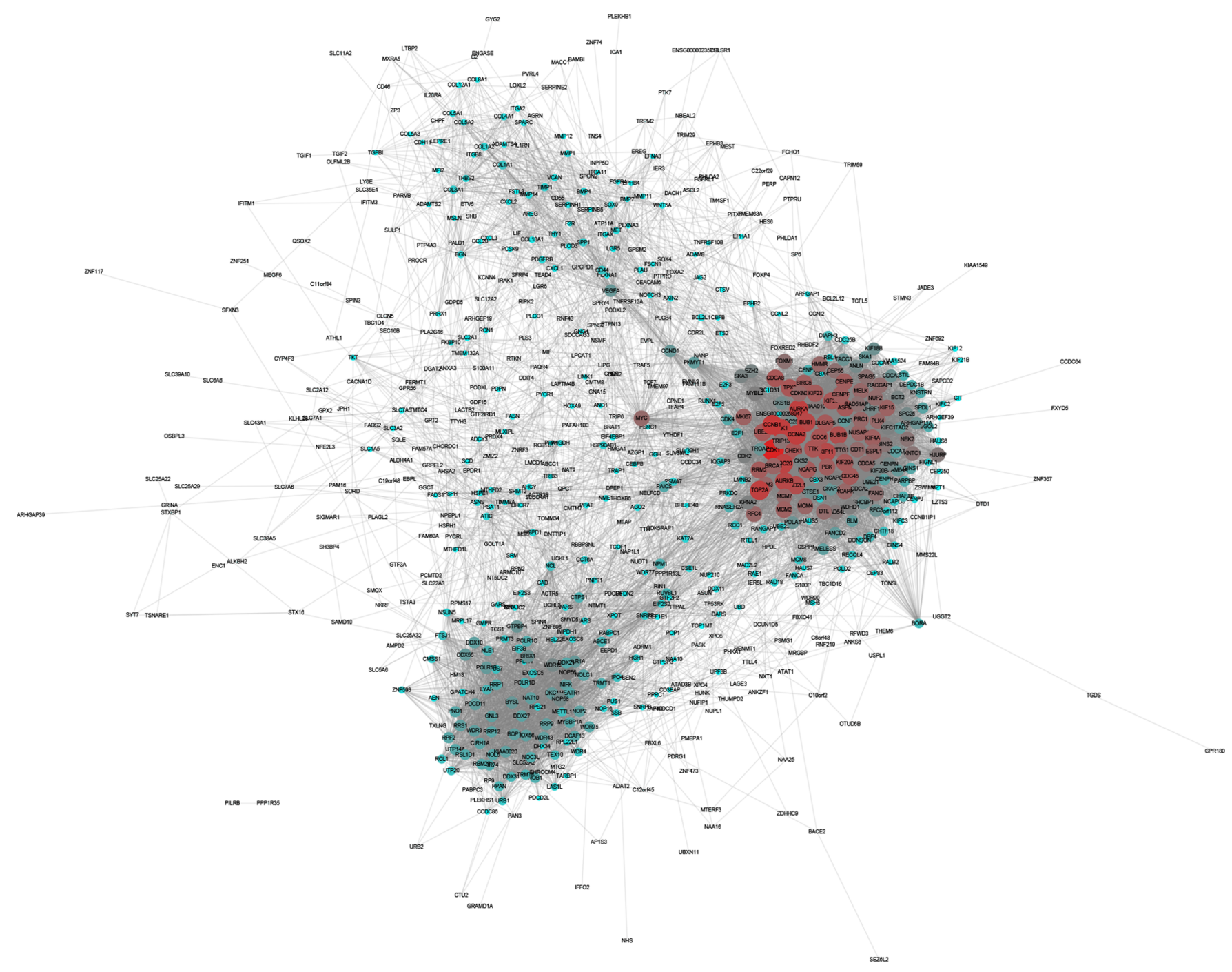

Figure 3. PPI network analysis of upregulated DEGs in colorectal cancer. The upregulated PPI network was composed of 649 nodes and 11,747 edges. Red indicates genes with more connections with other genes, blue indicates genes with less connections with other genes. PPI, protein-protein interaction.

process (Fig. 6A). TCGA analysis revealed that UBE2T RNA expression was upregulated in colon adenocarcinoma (COAD) (Fig. 7A) and rectum adenocarcinoma (READ) (Fig. 7B) samples compared with adjacent non-tumor tissues. Kaplan-Meier analysis revealed that high UBE2T expression was associated with shorter overall survival time in CRC, despite the difference is not significant enough ( $\mathrm{P}>0.05$; Fig. $7 \mathrm{C})$.

Knockdown of UBE2T inhibits CRC cell proliferation. To investigate the molecular functions of UBE2T in CRC, lentivirus-mediated knockdown of UBE2T in RKO cells was performed. RT-qPCR and western blotting demonstrated that both the RNA and protein expression levels of UBE2T were reduced in RKO cells infected with shUBE2T lentivirus compared with cells infected with control lentivirus (Fig. 7D and E). Furthermore, western blot analysis of Bcl-2 and cleaved caspase 3 and an Annexin V/FACS kit were used to detect cell apoptosis after knockdown of UBE2T in RKO cells. UBE2T knockdown induced a reduction in $\mathrm{Bcl}-2$ expression and an increase in cleaved caspase 3 protein expression in RKO cells (Fig. 7F). The percentage of apoptotic RKO cells was significantly increased in the UBE2T knockdown group compared with the control group (Fig. 7G and H). These results demonstrated that UBE2T was associated with the regulation of apoptosis in CRC.

To detect the effects of UBE2T knockdown on proliferation in RKO cells, a Celigo ${ }^{\circledR}$ assay was used to monitor cell proliferation for 5 days (Fig. 8A and B). Knockdown of UBE2T exerted a significant impairment on cell proliferation from day 3 compared with the normal group (Fig. 8A and B).

Knockdown of UBE2T induces cell cycle arrest. A colony formation assay was performed to validate the role of UBE2T in the regulation of CRC cell proliferation. The results demonstrated that UBE2T knockdown in RKO cells induced a decrease in colony formation compared with the control group (Fig. 8C and D). The number of colonies in the UBE2T knockdown group was reduced by $~ 50 \%$ compared with the control group (Fig. 8C and D).

The abnormal regulation of the cell cycle and apoptosis contributes to proliferation in cancer cells (24). To investigate the roles of UBE2T in the cell cycle and apoptosis, a series of flow cytometry assays was performed. Using a PI/FACS 


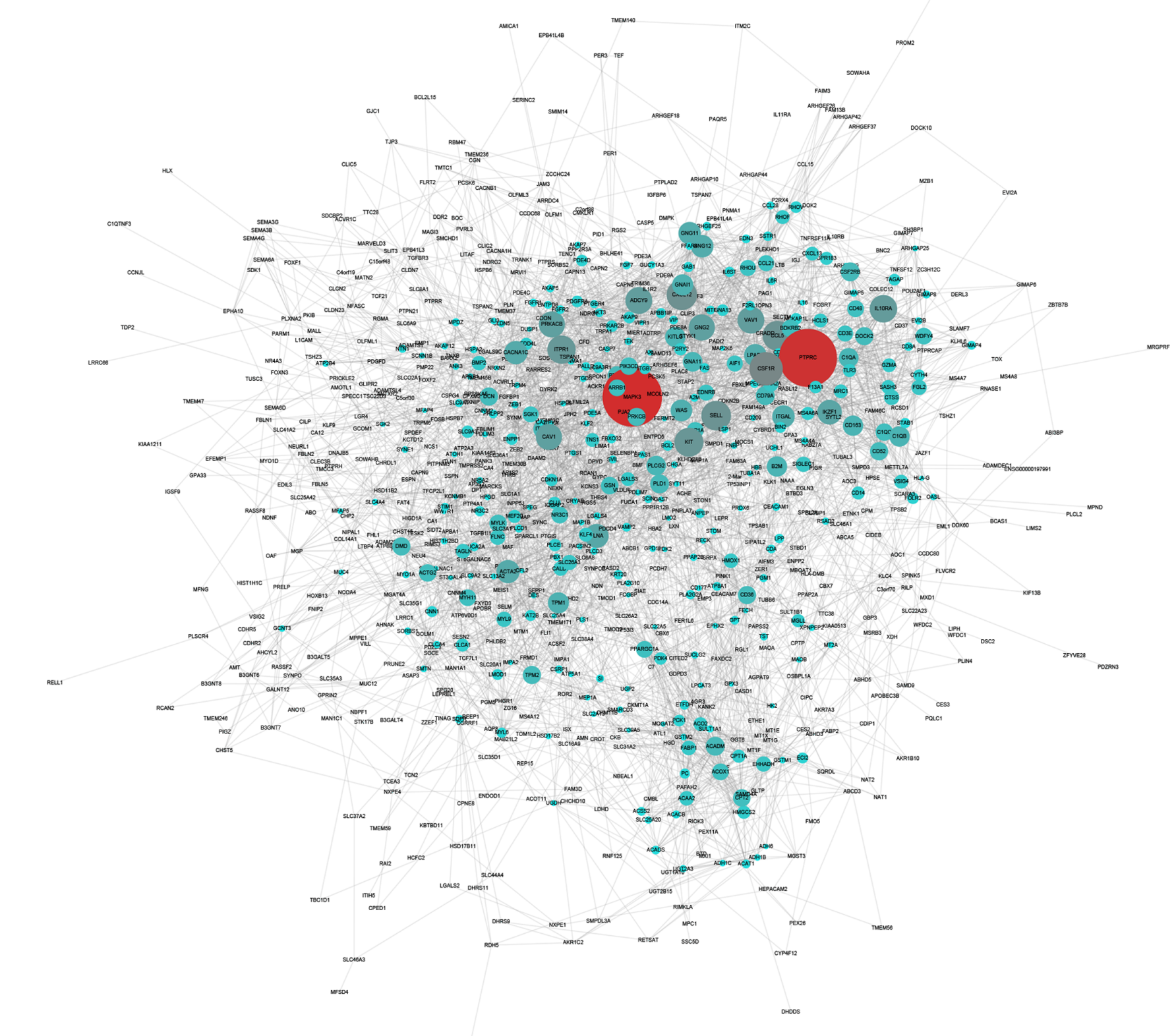

Figure 4. PPI network analysis of downregulated DEGs in colorectal cancer. The downregulated PPI network was composed of 771 nodes and 4,232 edges. Red indicates genes with more connections with other genes, blue indicates genes with less connections with other genes. PPI, protein-protein interaction.

kit, it was revealed that the percentage of cells in the $\mathrm{S}$ phase was significantly reduced in UBE2T knockdown RKO cells compared with the control group. However, the percentage of cells in the $\mathrm{G}_{1}$ phase was significantly increased in UBE2T knockdown RKO cells compared with the control group, suggesting that the UBE2T gene was associated with cell cycle regulation (Fig. 8E and F).

\section{Discussion}

Identification of key regulators that contribute to cancer progression is critical for cancer diagnosis and treatment. In the present study, 1,835 genes were identified to be DEGs, including 811 upregulated and 1,024 downregulated genes. Bioinformatics analysis revealed that these DEGs were associated with the regulation of cell proliferation of CRC by modulating multiple pathways, including mitotic nuclear division, response to radiation, Cell cycle, mitotic cell cycle process, mitotic sister chromatid segregation, cell proliferation and DNA metabolic process.

To identify hub genes in CRC, PPI networks were constructed and two hub networks were revealed. Among them, the upregulated hub network included 101 nodes and the downregulated hub network included 15 nodes. Most of these 


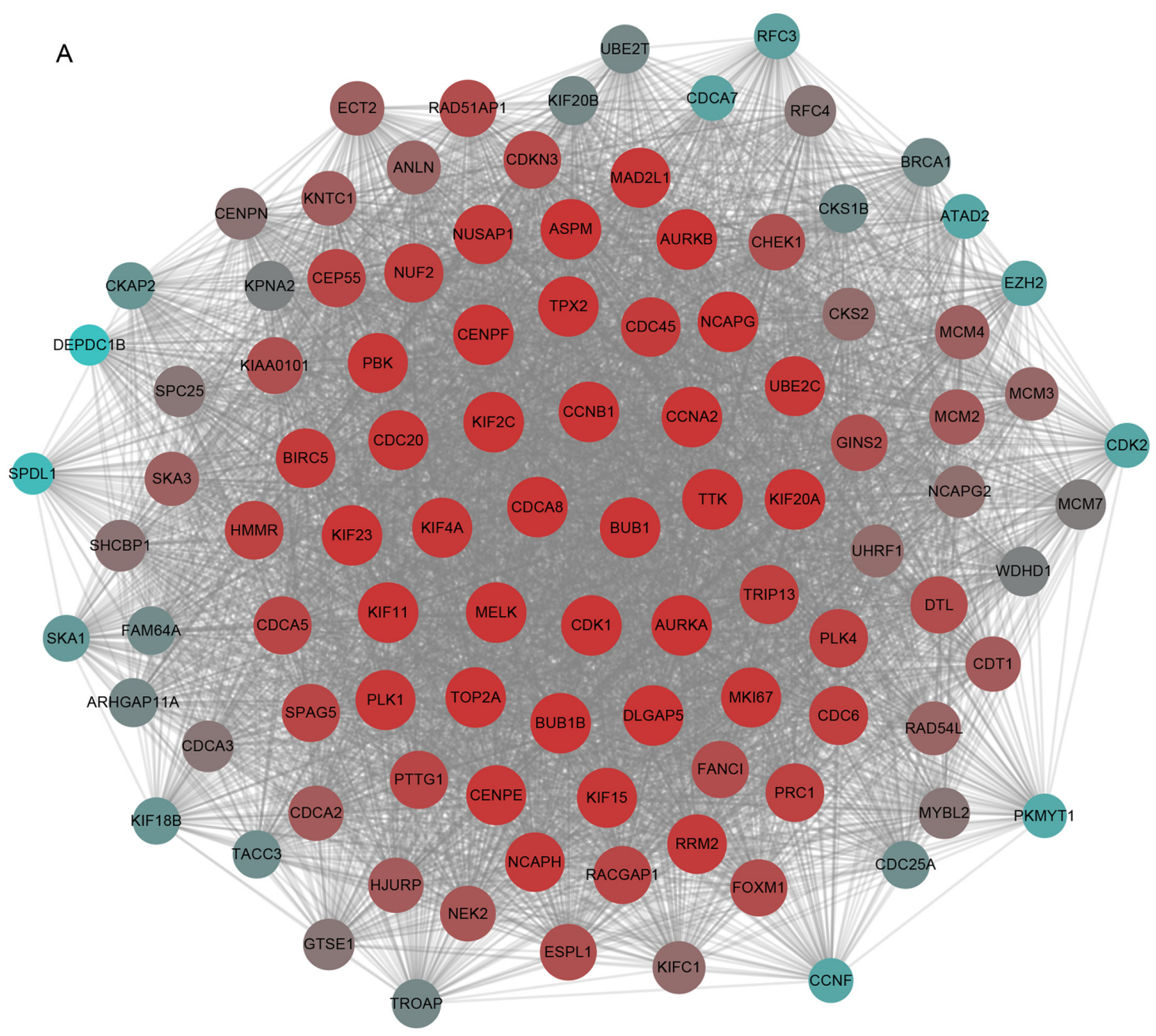

B

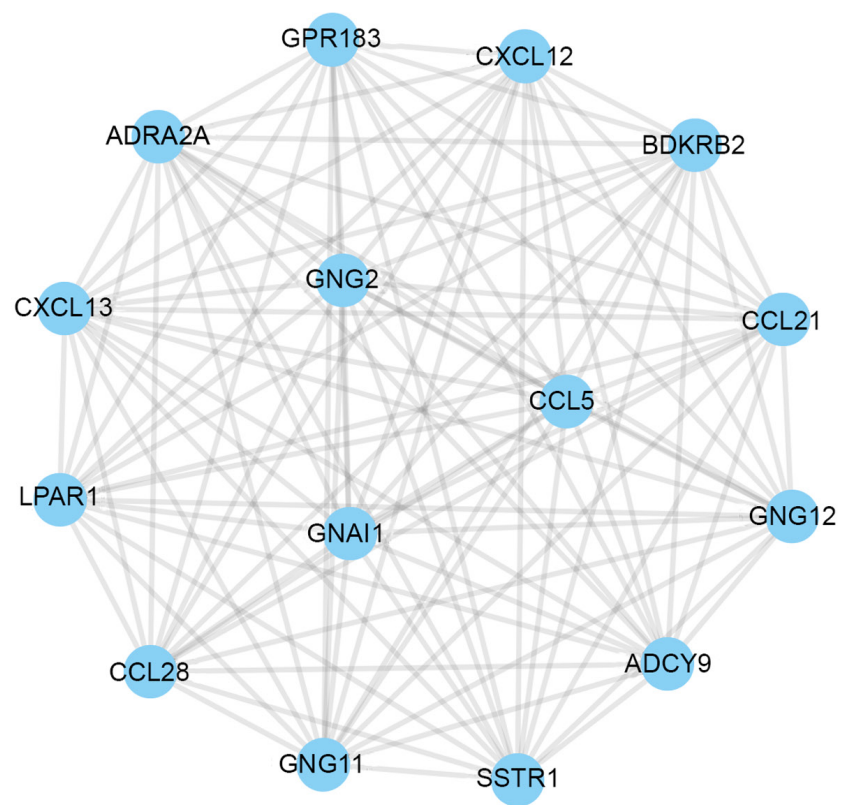

Figure 5. Hub protein-protein interaction network analysis of differentially expressed genes in colorectal cancer. (A) Hub upregulated network including 101 nodes and 4,765 edges. (B) Hub downregulated network including 15 nodes and 105 edges. Red indicates genes with more connections with other genes, blue indicates genes with less connections with other genes.

genes have been reported to serve a crucial role in human CRC (25-28). For example, high serine/threonine-protein kinase PLK4 expression promotes tumor progression and induces epithelial-mesenchymal transition by regulating the 


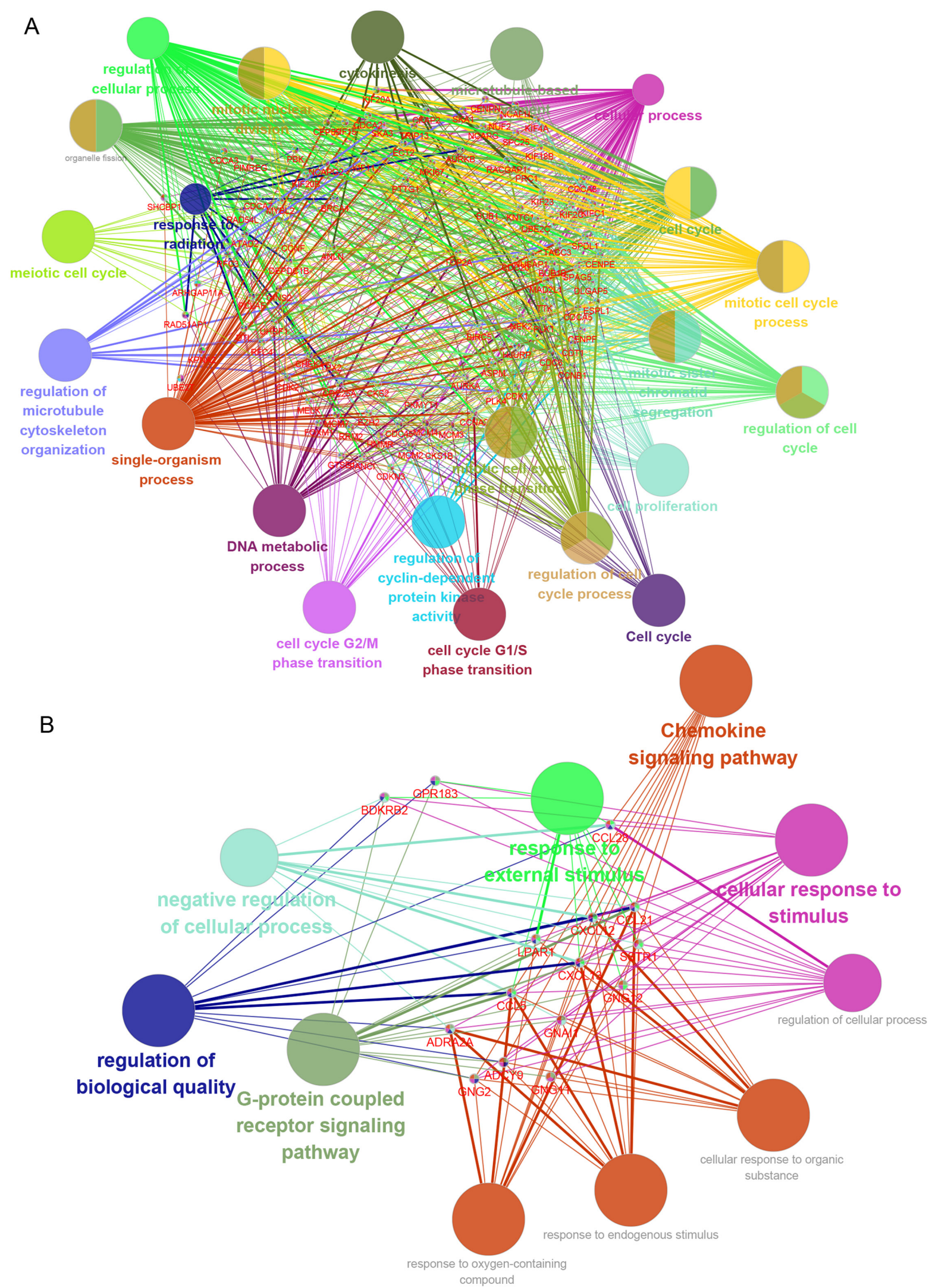

Figure 6. Functional annotation of hub protein-protein interaction networks in CRC. (A) Functional annotation of the hub upregulated network in CRC. (B) Functional annotation of the hub downregulated network in CRC. Different colors represent different biological processes, as indicated in the figure. CRC, colorectal cancer.

Wnt/ß-catenin signaling pathway in CRC (25). Serine/threonine-protein kinase PLK1 has tumor-suppressive potential in adenomatous polyposis coli-truncated colon cancer cells (26).
Cell division cycle-associated protein 3 mediates p21-dependent proliferation by regulating E2F transcription factor 1 expression in CRC (27). Mitotic checkpoint kinase monopolar 
A

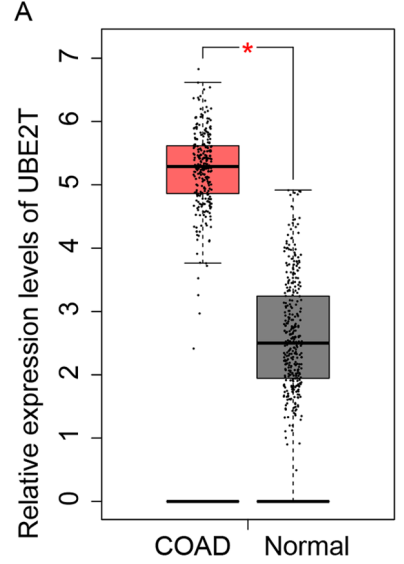

D

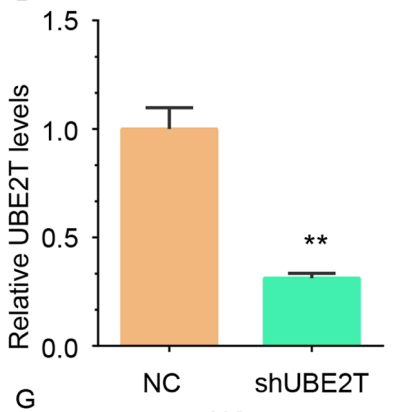

NC

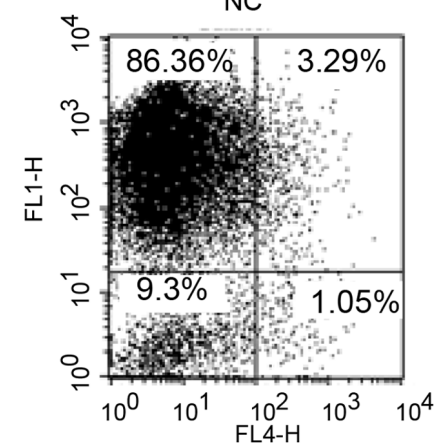

B

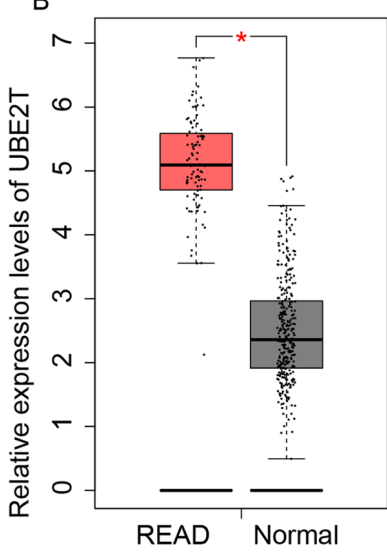

E

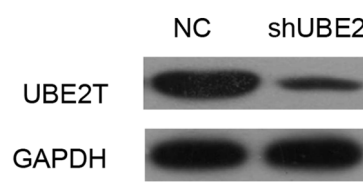

C

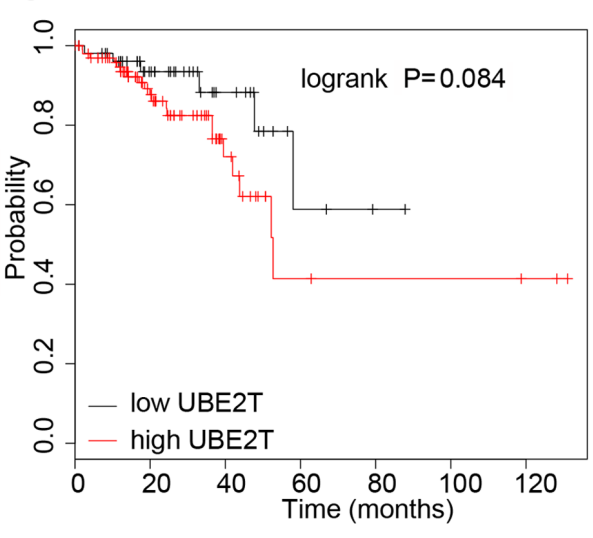

F

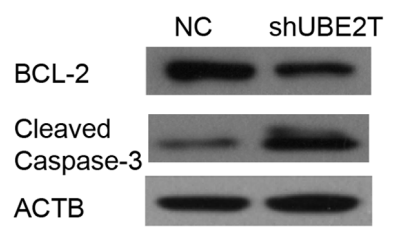

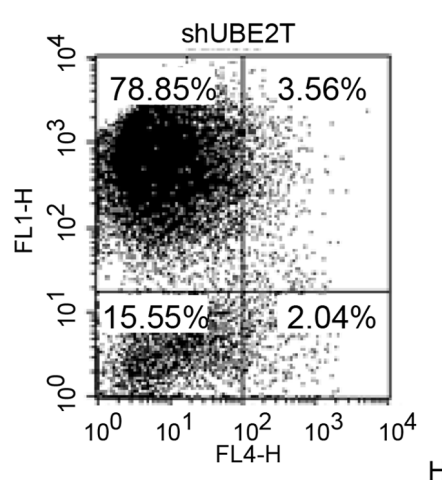

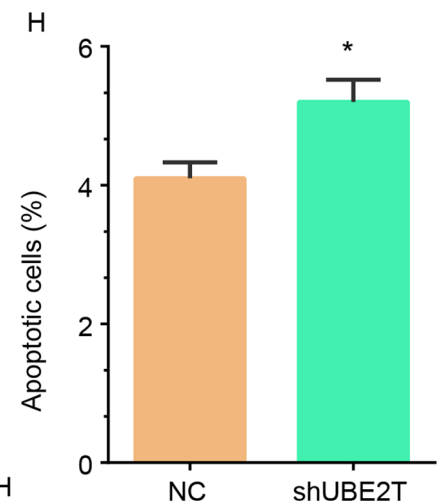

Figure 7. Knockdown of UBE2T suppresses CRC cell proliferation. UBE2T expression was upregulated in (A) COAD and (B) READ samples compared with matched normal tissues. (C) Higher expression levels of UBE2T were associated with shorter overall survival time compared to low UBE2T expression in patients with CRC. (D) RNA and (E) protein expression levels of UBE2T were reduced in RKO cells after transfection with siUBE2T compared with those of the control group (F) Bcl-2 protein expression was reduced and cleaved-caspase 3 expression was increased in RKO cells after transfection with siUBE2T compared with those in the control group. (G) Apoptotic rates of RKO cells were determined by flow cytometry. (H) Knockdown of UBE2T induces cell apoptosis of RKO cells compared with the control group. ${ }^{*} \mathrm{P}<0.05 ;{ }^{* *} \mathrm{P}<0.01$ vs. NC. COAD, colon adenocarcinoma; CRC, colorectal cancer; NC, negative control; READ, rectum adenocarcinoma; si, small interfering RNA; UBE2T, ubiquitin-conjugating enzyme E2 T.

spindle $1 /$ TTK protein kinase is associated with the prognosis of patients with colon cancer and regulates tumor cell proliferation and differentiation via the protein kinase $\mathrm{C} \alpha / \mathrm{ERK} 1 / 2$ and PI3K/Akt signaling pathways (29). PDZ binding kinase/T-lymphokine-activated killer cell-originated protein kinase interacts with the DNA-binding domain of tumor suppressor p53 and modulates the expression of transcriptional targets, including p21 (30).

Notably, the present study identified that multiple members of ubiquitin-conjugating enzyme E family served a key role in CRC, which have been reported to modulate the progression of human cancer, such as UBE2T (11) and UBE2C (31). In previous reports, ubiquitin-conjugating enzyme $\mathrm{E}$ family members have been identified to regulate cancer development $(32,33)$. For instance, UBE2C expression is higher in late-stage tumors compared with in early-stage tumors (32). Ubiquitin-conjugating enzyme UBE2O has been reported to regulate the cellular clock function by promoting the degradation of the transcription factor aryl hydrocarbon receptor nuclear translocator-like protein 1 (33). UBE2T is a member of the ubiquitinconjugating enzyme family. The ubiquitin-proteasome signaling pathway serves a key role in cell proliferation and DNA damage repair $(28,34,35)$. UBE2T expression has been reported to be upregulated in multiple types of tumors, such as prostate cancer and lung cancer $(6,8,9,11,12,36,37)$. However, to the best of our knowledge, the association between UBE2T and CRC has not been investigated. Furthermore, to the best of our knowledge, the present study was the first to demonstrate that UBE2T expression was upregulated in COAD and READ samples compared with normal tissues. 

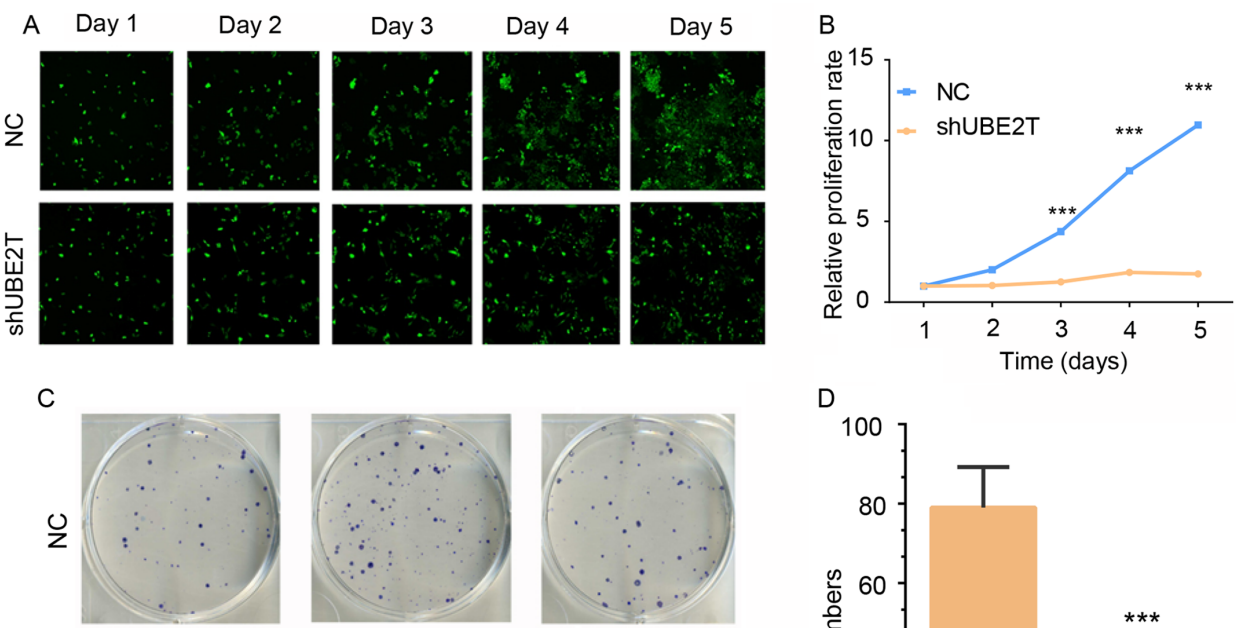

D
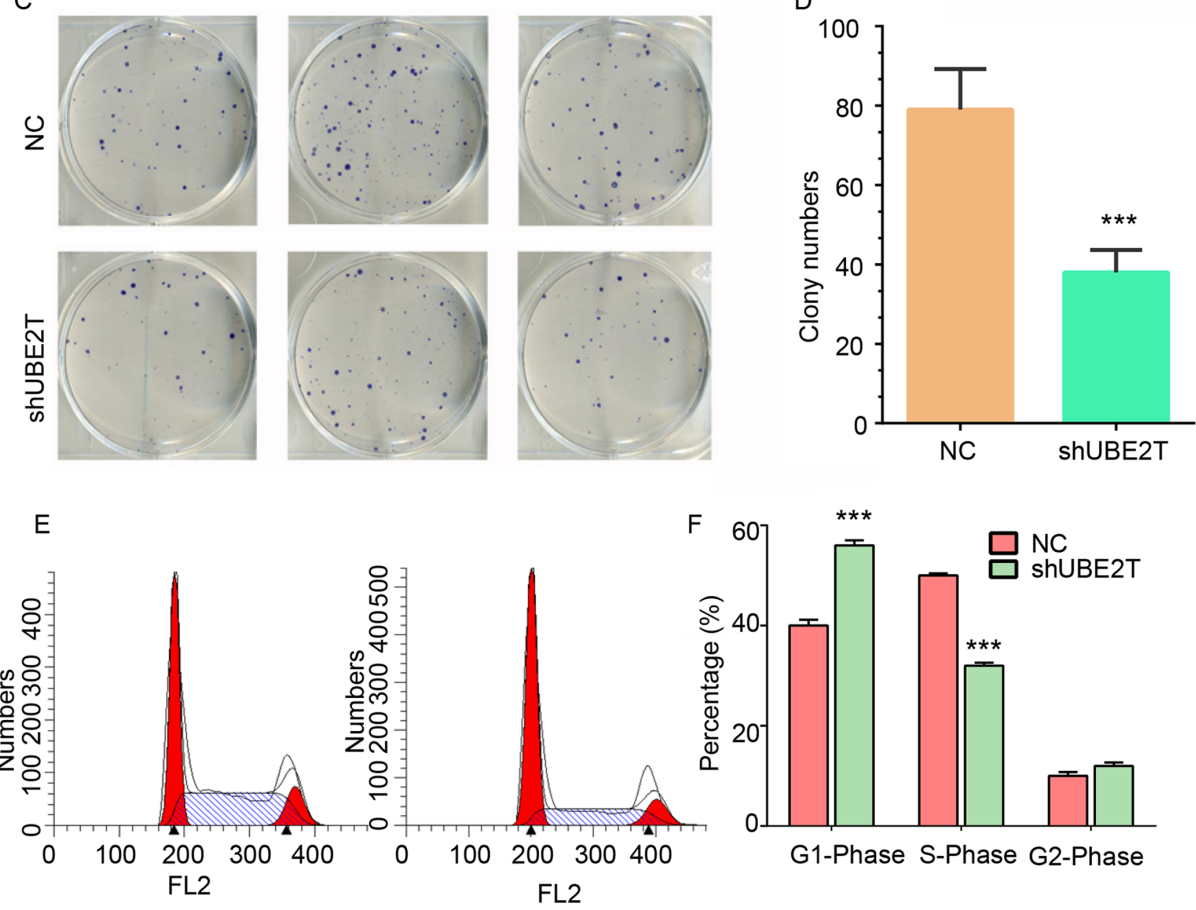

Figure 8. Knockdown of UBE2T suppresses colorectal cancer cell proliferation and colony formation but induces cell cycle arrest. (A) Celigo ${ }^{\circledR}$ assay revealing that the cell numbers in UBE2T-knockdown RKO cells were significantly reduced compared with the control group. Magnification, x100. (B) Quantification of the cell numbers in UBE2T knockdown RKO group and control group. (C) Colony formation assay showed Knockdown of UBE2T suppressed cell colony formation of RKO cells compared with the control group. (D) Quantification of the colony numbers after knockdown of UBE2T in RKO cells compared with the control group. (E) Flow cytometry assays showed cell cycle distribution of RKO cells after UBE2T knockdown. Y-axis represent cell numbers. (F) Knockdown of UBE2T induced cell cycle arrest of RKO cells compared with the control group. ${ }^{* * * *} \mathrm{P}<0.001 \mathrm{vs.} \mathrm{NC.} \mathrm{FL2,} \mathrm{orange-red} \mathrm{light} \mathrm{(564-606} \mathrm{nm)} \mathrm{for}$ FACScans and FACSCaliburs; NC, negative control; si, small interfering RNA; UBE2T, ubiquitin-conjugating enzyme E2 T.

Kaplan-Meier analysis revealed that higher levels of UBE2T were associated with worse prognosis compared with low UBE2T expression levels in CRC. Additionally, the present study revealed that knockdown of UBE2T inhibited CRC cell proliferation. Flow cytometry assays revealed that UBE2T knockdown induced cell cycle arrest and apoptosis in vitro.

In summary, the present study screened DEGs in CRC. Further validation demonstrated that UBE2T expression was upregulated in CRC and knockdown of UBE2T inhibited the proliferation of CRC cells by inducing cell cycle arrest and apoptosis. These results indicated that UBE2T may be a novel potential biomarker for CRC.

\section{Acknowledgements}

Not applicable.

\section{Funding}

No funding was received.

\section{Availability of data and materials}

The datasets used and/or analyzed during the current study are available from the corresponding author upon reasonable request.

\section{Authors' contributions}

ML and YZ designed the study and drafted the initial manuscript. ML performed the experiments. YZ analyzed the data. ML and YZ confirmed the authenticity of all the raw data. All authors read and approved the final version of the manuscript.

\section{Ethics approval and consent to participate}

Not applicable.

\section{Patient consent for publication}

Not applicable. 


\section{Competing interests}

The authors declare that they have no competing interests.

\section{References}

1. Rawla P, Sunkara T and Barsouk A: Epidemiology of colorectal cancer: Incidence, mortality, survival, and risk factors. Prz Gastroenterol 14: 89-103, 2019.

2. Siegel RL, Miller KD, Fedewa SA, Ahnen DJ, Meester RGS Barzi A and Jemal A: Colorectal cancer statistics, 2017. CA Cancer J Clin 67: 177-193, 2017.

3. Chen W, Zheng R, Baade PD, Zhang S, Zeng H, Bray F, Jemal A $\mathrm{Yu}$ XQ and He J: Cancer statistics in China, 2015. CA Cancer J Clin 66: 115-132, 2016

4. Morreale FE, Bortoluzzi A, Chaugule VK, Arkinson C Walden $\mathrm{H}$ and Ciulli $\mathrm{A}$ : Allosteric targeting of the fancon anemia ubiquitin-conjugating enzyme Ube2T by fragment screening. J Med Chem 60: 4093-4098, 2017.

5. Alpi A, Langevin F, Mosedale G, Machida YJ, Dutta A and Patel KJ: UBE2T, the Fanconi anemia core complex, and FANCD2 are recruited independently to chromatin: A basis for the regulation of FANCD2 monoubiquitination. Mol Cell Biol 27: 8421-8430, 2007

6. Liu LL, Zhu JM, Yu XN, Zhu HR, Shi X, Bilegsaikhan E, Guo HY, Wu J and Shen XZ: UBE2T promotes proliferation via G2/M checkpoint in hepatocellular carcinoma. Cancer Manag Res 11: 8359-8370, 2019.

7. Alpi AF, Pace PE, Babu MM and Patel KJ: Mechanistic insight into site-restricted monoubiquitination of FANCD2 by Ube2t, FANCL, and FANCI. Mol Cell 32: 767-777, 2008.

8. Hao J, Xu A, Xie X, Hao J, Tian T, Gao S, Xiao X and He D: Elevated expression of UBE2T in lung cancer tumors and cell lines. Tumour Biol 29: 195-203, 2008.

9. Hao P, Kang B, Li Y, Hao W and Ma F: UBE2T promotes proliferation and regulates PI3K/Akt signaling in renal cell carcinoma. Mol Med Rep 20: 1212-1220, 2019.

10. Liu LP, Yang M, Peng QZ, Li MY, Zhang YS, Guo YH, Chen Y and Bao SY: UBE2T promotes hepatocellular carcinoma cell growth via ubiquitination of p53. Biochem Biophys Res Commun 493: 20-27, 2017

11. Luo C, Yao Y, Yu Z, Zhou H, Guo L, Zhang J, Cao H, Zhang G, Li Y and Jiao Z: UBE2T knockdown inhibits gastric cancer progression. Oncotarget 8: 32639-32654, 2017.

12. Wen M, Kwon Y, Wang Y, Mao JH and Wei G: Elevated expression of UBE2T exhibits oncogenic properties in human prostate cancer. Oncotarget 6: 25226-25239, 2015.

13. Huang da W, Sherman BT and Lempicki RA: Systematic and integrative analysis of large gene lists using DAVID bioinformatics resources. Nat Protoc 4: 44-57, 2009.

14. Huang DW, Sherman BT, Tan Q, Kir J, Liu D, Bryant D, Guo Y, Stephens R, Baseler MW, Lane HC and Lempicki RA: DAVID bioinformatics resources: Expanded annotation database and novel algorithms to better extract biology from large gene lists. Nucleic Acids Res 35: W169-W175, 2007.

15. Szklarczyk D, Franceschini A, Wyder S, Forslund K, Heller D, Huerta-Cepas J, Simonovic M, Roth A, Santos A, Tsafou KP, et al: STRING v10: Protein-protein interaction networks, integrated over the tree of life. Nucleic Acids Res 43: D447-D452, 2015.

16. Shannon P, Markiel A, Ozier O, Baliga NS, Wang JT, Ramage D, Amin N, Schwikowski B and Ideker T: Cytoscape: A software environment for integrated models of biomolecular interaction networks. Genome Res 13: 2498-2504, 2003.

17. Bader GD and Hogue CW: An automated method for finding molecular complexes in large protein interaction networks. BMC Bioinformatics 4: 2, 2003

18. Shi J, Zhong X, Song Y, Wu Z, Gao P, Zhao J, Sun J, Wang J, Liu J and Wang Z: Long non-coding RNA RUNX1-IT1 plays a tumour-suppressive role in colorectal cancer by inhibiting cell proliferation and migration. Cell Biochem Funct 37: 11-20, 2019

19. Livak KJ and Schmittgen TD: Analysis of relative gene expression data using real-time quantitative PCR and the 2(-Delta Delta C(T)) method. Methods 25: 402-408, 2001
20. Chuang KC, Chen FW, Tsai MH and Shieh JJ: EGR-1 plays a protective role in AMPK inhibitor compound C-induced apoptosis through ROS-induced ERK activation in skin cancer cells. Oncol Lett 21: 304, 2021.

21. Cui F, Hu J, Ning S, Tan J and Tang H: Overexpression of MCM10 promotes cell proliferation and predicts poor prognosis in prostate cancer. Prostate 78: 1299-1310, 2018.

22. Kelsall IR, Langenick J, MacKay C, Patel KJ and Alpi AF The Fanconi anaemia components UBE2T and FANCM are functionally linked to nucleotide excision repair. PLoS One 7: e36970, 2012

23. Rickman KA, Lach FP, Abhyankar A, Donovan FX, Sanborn EM, Kennedy JA, Sougnez C, Gabriel SB, Elemento O, Chandrasekharappa SC, et al: Deficiency of UBE2T, the E2 ubiquitin ligase necessary for FANCD2 and FANCI ubiquitination, causes FA-T subtype of fanconi anemia. Cell Rep 12: 35-41, 2015.

24. Seoane J: Cancer: Division hierarchy leads to cell heterogeneity. Nature 549: 164-166, 2017.

25. Liao Z, Zhang H, Fan P, Huang Q, Dong K, Qi Y, Song J, Chen L, Liang H, Chen X, et al: High PLK4 expression promotes tumor progression and induces epithelial-mesenchymal transition by regulating the $\mathrm{Wnt} / \beta$-catenin signaling pathway in colorectal cancer. Int J Oncol 54: 479-490, 2019.

26. Raab M, Sanhaji M, Matthess Y, Hörlin A, Lorenz I, Dötsch C, Habbe N, Waidmann O, Kurunci-Csacsko E, Firestein R, et al: PLK1 has tumor-suppressive potential in APC-truncated colon cancer cells. Nat Commun 9: 1106, 2018.

27. Qian W, Zhang Z, Peng W, Li J, Gu Q, Ji D, Wang Q, Zhang Y, Ji B, Wang S, et al: CDCA3 mediates p21-dependent proliferation by regulating E2F1 expression in colorectal cancer. Int J Oncol 53: 2021-2033, 2018.

28. Nakayama KI and Nakayama K: Ubiquitin ligases: Cell-cycle control and cancer. Nat Rev Cancer 6: 369-381, 2006.

29. Zhang L, Jiang B, Zhu N, Tao M, Jun Y, Chen X, Wang Q and Luo C: Mitotic checkpoint kinase Mps1/TTK predicts prognosis of colon cancer patients and regulates tumor proliferation and differentiation via PKC $\alpha /$ ERK1/2 and PI3K/Akt pathway. Med Oncol 37: 5, 2019.

30. Hu F, Gartenhaus RB, Eichberg D, Liu Z, Fang HB and Rapoport AP: PBK/TOPK interacts with the DBD domain of tumor suppressor p53 and modulates expression of transcriptional targets including p21. Oncogene 29: 5464-5474, 2010

31. Zhang HQ, Zhao G, Ke B, Ma G, Liu GL, Liang H, Liu LR and Hao XS: Overexpression of UBE2C correlates with poor prognosis in gastric cancer patients. Eur Rev Med Pharmacol Sci 22: 1665-1671, 2018.

32. Dastsooz H, Cereda M, Donna D and Oliviero S: A comprehensive bioinformatics analysis of UBE2C in cancers. Int J Mol Sci 20: 2228, 2019.

33. Chen S, Yang J, Zhang Y, Duan C, Liu Q, Huang Z, Xu Y, Zhou L and $\mathrm{Xu} \mathrm{G}$ : Ubiquitin-conjugating enzyme UBE2O regulates cellular clock function by promoting the degradation of the transcription factor BMAL1. J Biol Chem 293: 11296-11309, 2018.

34. Voutsadakis IA: Ubiquitin- and ubiquitin-like proteins-conjugating enzymes (E2s) in breast cancer. Mol Biol Rep 40: 2019-2034, 2013.

35. Hoeller D, Hecker CM and Dikic I: Ubiquitin and ubiquitin-like proteins in cancer pathogenesis. Nat Rev Cancer 6: 776-788, 2006.

36. Zhang W, Zhang Y, Yang Z, Liu X, Yang P, Wang J, Hu K, He X, Zhang X and Jing H: High expression of UBE2T predicts poor prognosis and survival in multiple myeloma. Cancer Gene Ther 26: 347-355, 2019.

37. Gong YQ, Peng D, Ning XH, Yang XY, Li XS, Zhou LQ and Guo YL: UBE2T silencing suppresses proliferation and induces cell cycle arrest and apoptosis in bladder cancer cells. Oncol Lett 12: 4485-4492, 2016.

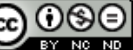

This work is licensed under a Creative Commons Attribution-NonCommercial-NoDerivatives 4.0 International (CC BY-NC-ND 4.0) License. 